\title{
Mujeres en órganos de gobierno universitarios. Nuevo contexto normativo y políticas de igualdad
}

\author{
Women at University top management levels. \\ New legal context and equity plans
}

\author{
María del Carmen Díaz-Fernández / cardiaz@us.es \\ orcid.org/0000-0001-8203-2150 \\ María del Rocío Martínez-Torres / rmtorres@us.es \\ orcid.org/0000-0002-1640-0020 \\ Jesús Manuel López-Bonilla / lopezbon@us.es \\ orcid.org/0000-0003-0623-1048 \\ Universidad de Sevilla, España
}

\begin{abstract}
This research aims to identify the main barriers that make the access of women to top management levels in Universities difficult. These barriers promote the generally known and assumed reality of gender inequality in these governing bodies. A scientific method, Concept Mapping (Trochim, 1989) has been used to this aim. This methodology, whose use is new in this field of research, has allowed us to identify both the causes and possible solutions. Results highlight the need to undertake strategic lines in policies of equality, focusing on eliminating certain weaknesses and threats detected, in order to achieve effective gender equality. These results, in turn, can also be considered as a starting point in the design of real and effective equality governmental university gender policies in many countries.
\end{abstract}

Key words: woman, gender, equities plans, universities' government, public administration.

Resumen: El propósito de este trabajo ha sido identificar las principales barreras que dificultan el acceso equitativo de las mujeres a los niveles directivos superiores en las universidades españolas, favoreciendo una realidad generalmente conocida y asumida de desigualdad de género en estos órganos de gobierno. Para ello se ha empleado la técnica científica de elaboración de mapas conceptuales (Trochim, 1989). El empleo de esta metodología, innovadora en su aplicación en esta área, nos ha permitido conocer tanto las causas como una serie de posibles soluciones a la problemática tratada. Así pues, los resultados del trabajo ponen de relieve la necesidad de acometer las líneas estratégicas en las distintas políticas de igualdad, en las que se haga especial énfasis en la subsanación de determinadas debilidades y amenazas, detectadas a partir del empleo de la metodología utilizada, en aras del logro de la igualdad efectiva de género. Unos resultados que, a su vez, pueden ser considerados como punto de partida para el diseño de políticas de igualdad real y efectiva en el ámbito del gobierno de universidades públicas y privadas de muchos países.

Palabras clave: mujer, género, políticas de igualdad, órganos de gobierno universitario, administración pública. 


\section{Introducción ${ }^{1}$}

Numerosos estudios han encontrado evidencias en cuanto a los obstáculos que más allá de su día a día, en la sociedad (Maldonado, 2003), dificultan e impiden el desarrollo y promoción de las mujeres en la misma, especialmente en el mundo laboral. "La economía feminista hace una contribución extensa al estudio de la participación económica de las mujeres, en particular revelando los mecanismos de discriminación en el mercado laboral" (Rodríguez, 2015: 34).

La preocupación en este sentido, tanto a nivel social, como político y empresarial, es cada vez mayor, a la par de las críticas, las muestras de rechazo a la misma y las acciones tendentes a encontrarles una solución (Rees, 2001; Subramaniam, 2003; Lambert y Parker, 2006; Priola, 2007; Clegg, 2008; Timmers et al., 2010). Estas circunstancias han dado lugar, por un lado, a un incremento en el número de trabajos que tratan de ahondar en esta situación desfavorecedora de la mujer frente al hombre, ofreciendo numerosas explicaciones a esta infrarrepresentación y a la desigualdad de género que conlleva (Phelps, 1972; Pearce y Zahra, 1992; Frundt, 2002; Wolfers, 2006; Grueso, 2010; Salinas et al., 2010; Palma y Cerva, 2014; López et al., 2014).

Y por otro lado, a la consecución de grandes logros. En este sentido, "el concepto de género como categoría social de análisis es una de las contribuciones teóricas más significativas del feminismo contemporáneo. Surgió para explicar las desigualdades entre varones y mujeres, y para dar cuenta de cómo la noción de lo femenino y lo masculino se conforma a partir de una relación mutua, cultural e histórica" (Rodríguez, 2015:31).

A pesar de todos estos avances, sociales, políticos-legales y de los logros alcanzados, la desigualdad de género es un tema todavía presente en muchos países, tanto a nivel social, como empresarial (García, 2008). Es más, esta desigualdad parece persistir también en instituciones públicas como la universitaria (Broadbridge y Fielden, 2015).

Tradicionalmente, tanto a nivel político como académico, el interés por la desigualdad de género ha estado más centrado en las grandes empresas privadas. Empresas en las que este fenómeno discriminatorio ha estado más presente y con mayor intensidad, especialmente en el camino de la mujer

1 El presente estudio ha sido financiado por el Ministerio de Educación y Ciencia, España. Proyecto de investigación EA2009-0039: Análisis del acceso de la mujer a los puestos directivos de las universidades españolas y propuestas para conseguir la igualdad participativa de género. 
hacia la alta dirección (de Cabo et al., 2010). "La posición de la mujer en la dirección ha sido un tema de discusión largamente discutido" (Broadbridge y Fielden, 2015: 1); existe una amplia literatura en torno a la persistencia de barreras que impiden el desarrollo profesional de las mujeres universitarias. Sus consecuencias quedan patentes no sólo en los momentos clave de este proceso, sino que actúan significativamente en la consolidación del mismo (Donoso et al., 2011).

En la vida universitaria, la mujer se enfrenta tanto a lastres "convencionales", estrechamente vinculados a factores culturales y sociales (techos de cristal y de cemento) (Morrison et al., 1987; Eisenmann, 2003; Probert, 2005; Arreman y Weiner, 2007; Gisbert, 2007; Jalalzai, 2008), como a lastres estructurales, derivados de su tardía incorporación a la vida profesional y educativa. Se podría decir, pues, que no es su condición femenina el impedimento para ejercer, sino, más bien, un condicionante para que aún no todas alcancen hacerlo (Lovell, 2006).

La mayor presencia en las aulas y los mejores resultados académicos de las mujeres en comparación con sus homónimos (Marcenaro et al., 2007) no parecen contribuir al logro de la equidad entre géneros tan largamente reclamada por las mujeres. El hecho de que esta mayor presencia femenina parezca estar concentrada en determinadas áreas como Ciencias Sociales, Farmacia, Psicología, Pedagogía, Magisterio, "tradicionalmente atribuibles a su condición” (Guerrero, 2007), y que su acceso a los cuerpos docentes y a los órganos de gobierno sea aún en número reducido, parece contrarrestar el efecto positivo anterior.

Al centrarnos en la universidad española, encontramos además cómo la formación de su profesorado ha estado caracterizada, junto con un desarrollo temporal desigual a favor de los hombres, por currículos diferenciados en los cuales prima la formación moral y las "labores propias de su género" en las profesoras, y lo epistemológico y vocacional en los profesores (Guerrero, 2007).

En línea con estos argumentos, Alonso-Sánchez (2002: 469 y ss) resaltaba el carácter desigual de las trayectorias profesionales, y consecuentemente, de la situación de las mujeres en la universidad. Así, en su investigación se pone de manifiesto cómo las mujeres se estancan en las categorías más bajas del profesorado (ayudantes o asociados), encontrando numerosos obstáculos a la hora de acceder a posiciones que implican poder y privilegio en la universidad en contraste con los varones.

Esta situación -descrita por Sánchez en 2002-, parece hasta hoy en día, en 2017, no haber cambiado todavía en gran medida. Tal y como apuntaba la misma investigadora, esta asimetría profesional difícilmente 
será superada con el mero paso del tiempo, debido a que el origen de la desigualdad no es de naturaleza personal o de socialización profesional, sino de carácter institucional; todo se reduce a estar bien situado en los círculos de poder o no estarlo.

En este sentido el hombre tiene una fuerte y clara ventaja frente a la mujer. Y es que, como apuntaba Villavicencio (2014: 609) en su trabajo, "los derechos ideados para un 'ciudadano normal' no se acomodan a las necesidades de grupos diferenciados, pues demandan algo más que las tradicionales políticas distributivas del Estado democrático de derecho".

Afortunadamente, la segregación ocupacional está muy enquistada en la actualidad, al encontrarse su superación relacionada tanto con el aumento de los requisitos formativos y la mayor categoría ocupacional de los puestos de trabajo, como con el acceso universalista a "lo público", lo cual parece estar favoreciendo la entrada femenina a los mundos masculinizados (Ibáñez, 2010).

La igualdad entre mujeres y hombres pasa a ser un principio fundamental, basado en la participación activa y equilibrada de mujeres y hombres en todos los ámbitos de la vida; es decir, en la esfera civil, laboral, económica, social y cultural (Instituto Andaluz de la Mujer, 2011). En consonancia con lo anterior,

la economía feminista tiene como preocupación central la cuestión distributiva. $Y$ en particular, se concentra en reconocer, identificar, analizar y proponer cómo modificar la desigualdad de género como elemento necesario para lograr la equidad socioeconómica. En este sentido, la economía feminista es un programa académico pero también político. No tiene una pretensión aséptica de describir la realidad (como aquella que se atribuyen los economistas neoclásicos), sino un objetivo político de transformarla en un sentido más igualitario (Rodríguez, 2015: 32).

Institucionalizar el enfoque de género mediante estrategias en la Administración Pública es primordial. Provee de conceptos y objetivos a los planes, así como de conceptos y estrategias a los programas y proyectos que se ejecutan. Cimientos sobre los que se construye una estrategia eficiente en el proceso de planificación o diseño de acciones sociales. Y es que, en la medida en que las políticas sean correctamente formuladas, su desarrollo en otros instrumentos de menor jerarquía que orientan las acciones será más eficiente (García, 2003).

El presente trabajo, precedido por un estudio longitudinal de la presencia femenina en los distintos colectivos de las universidades españolas y en la composición de sus órganos unipersonales y colegiados (Martínez-Torres et al., 2010a), identifica -sirviéndose para ello de una técnica científica de fia- 
bilidad ampliamente reconocida- los mapas conceptuales (Trochim, 1983), las principales barreras que obstaculizan el acceso de las mujeres a los niveles directivos superiores en las universidades españolas.

Desde una perspectiva de género, los resultados alcanzados permiten señalar líneas estratégicas a tener en cuenta en el diseño de las políticas de igualdad universitarias, para promover el acceso equitativo entre mujeres y hombres; estos resultados pueden servir como punto de partida en el diseño de políticas de igualdad universitaria, que promuevan la tan largamente deseada y perseguida igualdad de género entre mujeres y hombres recogida desde los trabajos clásicos (Bourdieu, 2000), hasta otros más recientes (RodríguezEnríquez, 2015; Broadbridge y Fielden, 2015).

En las últimas décadas los estudios de género han contribuido a desvelar y a conocer ámbitos de la realidad hasta entonces inexplorados, reduciendo sesgos y errores en conceptos y teorías, y contribuyendo, incluso, a importantes reformulaciones disciplinares de algunos campos del conocimiento como ciencias sociales y humanidades (Ministerio de Ciencia e Innovación, 2011). La subsanación de estos sesgos, errores, barreras y desigualdades, así como el logro del acceso y promoción igualitaria de la mujer a los órganos de decisión de las instituciones públicas mediante políticas idóneas, justificarían la importancia de nuestro trabajo y de los resultados alcanzados.

El presente documento se estructura de la siguiente manera. A continuación, se recoge un análisis de las políticas de igualdad desarrolladas en las universidades españolas. Tras ello, se describe la metodología seguida para la consecución de nuestro objeto de estudio. Posteriormente, se presentan los resultados obtenidos, y finalmente, las principales conclusiones.

\section{Análisis de las políticas de igualdad en las distintas universidades españolas}

El estudio de la situación y posición de las mujeres en las políticas públicas es un tema de estudio y debate que se lleva a cabo desde hace más de tres décadas, a nivel nacional e internacional. Durante todo este tiempo han surgido paradigmas que han intentado explicar las desigualdades de género, aun cuando no siempre se tuvo claridad sobre el hecho de que tales desigualdades tenían raíces estructurales (García, 2008; Palma y Cerva, 2014; Archenti y Albaine, 2014; Ordorica-Mellado, 2014).

Tradicionalmente, se ha impuesto el dominio del hombre frente a la mujer.

La fuerza del orden masculino se descubre en el hecho de que prescinde de cualquier justificación: la visión androcéntrica se impone como neutra y no siente la necesidad de 
enunciarse en unos discursos capaces de legitimarla. El orden social funciona como una inmensa máquina simbólica que tiende a ratificar la dominación masculina en la que se apoya: es la división sexual del trabajo, distribución muy estricta de las actividades asignadas a cada uno de los dos sexos, de su espacio, su momento, sus instrumentos; es la estructura del espacio, con la oposición entre el lugar de reunión o el mercado, reservados a los hombres, y la casa, reservada a las mujeres [...]; es la estructura del tiempo, jornada, año agrario [...] masculinos, y los largos períodos de gestación, femeninos (Bourdieu, 2000: 11).

Es así como, la mujer, a lo largo del tiempo, por su condición femenina, se ha tenido que enfrentar a lo que podríamos denominar "lastres históricos" (dominio del hombre, economía y redes del cuidado, presión social, techo de cristal, techo de cemento...) (Rodríguez, 2015), lo cual ha dado lugar a que durante muchos años no sólo la incorporación de la mujer al mundo laboral fuese más tardía, sino que, de cara a esta incorporación, "fuese más probable que la mujer tuviera una cualificación más terciaria que los hombres en la mayoría de los países" (Broadbridge y Fielden, 2015: 1).

Contra estas fuerzas históricas, Bourdieu (2000) aboga por "una movilización típicamente política que abriría a las mujeres la posibilidad de una acción colectiva de resistencia, orientada hacia unas reformas jurídicas y políticas [...], opuesta a la resignación que estimula todas las visiones esencialistas (biologistas y psicoanalíticas) de la diferencia entre los sexos" (Bourdieu, 2000: 3).

Es más, defiende que hay que "convocar a las mujeres a comprometerse en una acción política, [...] en unas formas de organización y de acción colectivas y unas armas eficaces, simbólicas especialmente, capaces de quebrantar las instituciones, estatales y jurídicas, que contribuyen a eternizar su subordinación" (Bourdieu, 2000: 4).

Con el compromiso de transformar esta realidad de desigualdad entre los géneros y enfocando el problema desde distintos ámbitos, se han llevado a cabo desde entonces una serie de normas y políticas públicas orientadas a acabar con la desigualdad de oportunidades para mujeres y hombres. Esta regulación, fundamentada en el principio de presencia equilibrada o composición equilibrada, trata así de garantizar la igualdad en órganos y cargos de responsabilidad de las instituciones.

Esta nueva orientación y visión femenina -nueva línea de acción de la mujer en contra de la desigualdad de género- ha conseguido importantes logros en relación con su homónimo. Así, "si bien en el año 2000, los hombres superaban en número a las mujeres en los puestos directivos, en el 2011, la situación era la inversa" (Broadbridge y Fielden, 2015: 1). 
Del mismo modo, a partir de estos años, no sólo es más notorio el acceso de la mujer a la universidad, sino que, en la mayoría, su presencia en las aulas sobresale frente a la de los hombres. En cuanto a su cualificación, es destacable que los ratios se han invertido durante este espacio temporal de igual forma a favor de la mujer en cuanto al número de licenciadas, doctoras y a la mayor cualificación alcanzada por ella (Martín y Díaz, 2010), incrementando su valor como recurso para las empresas, no sólo como trabajadoras sino como futuras gestoras de las mismas. Por lo tanto, "cabría esperar que su posición en las jerarquías de gestión hubieran cambiado para reflejar esta nueva situación femenina" (Broadbridge y Fielden, 2015: 1).

Si nos centramos en el ámbito de la educación superior y ceñimos a las universidades españolas, el diagnóstico realizado por Martínez-Torres et al. (2010a) refleja el desequilibrio existente, tanto entre la presencia femenina en el sistema educativo, como en su representación en los órganos de decisión universitarios. Para hacerle frente y al amparo de textos legales de vital importancia como la Ley Orgánica de Modificación de la Ley Orgánica de Universidades (LOMLOU, 2007), se establecen medidas que se concretan en actuaciones específicas a desarrollar.

Un ejemplo de ello son las recogidas en el Plan Estratégico de Igualdad de Oportunidades (PEIO) 2014-2016. "Este Plan es el instrumento a través del cual el Gobierno de España define, en aquellos ámbitos que son competencia del Estado, los objetivos y medidas prioritarios para eliminar cualquier discriminación por razón de sexo que pueda persistir y para alcanzar la igualdad de oportunidades entre mujeres y hombres" (PEIO, 2014-2016: 8).

Así, este PEIO "responde al compromiso del Gobierno del Reino de España con la igualdad efectiva de mujeres y hombres" (PEIO, 2014-2016: 7). En el caso de la enseñanza superior, concretamente en el apartado denominado Eje 4, en el cual, haciéndose especial mención al ámbito específico de las universidades, en este plan se diseñan actuaciones que tratan de impulsar la igualdad de oportunidades de las mujeres en todos sus colectivos: Personal docente e investigador (PDI), Personal de administración y servicios (PAS) y Alumnado, además de la conciliación de su vida profesional, personal y familiar.

En este sentido, se impulsa el desarrollo en igualdad de la presencia de las mujeres en los Órganos de Gobierno, el apoyo a la creación de observatorios y comisiones de igualdad y el nombramiento de mujeres como Doctoras Honoris Causa. También se hace una referencia especial a las mujeres jóvenes en la universidad, fijando como medida el apoyo al asociacionismo de alumnas, potenciando la creación de una red de especialistas en los 
Estudios de Mujeres, Feministas y del Género, y con la creación de bases de datos de jóvenes investigadoras, superando de este modo el alejamiento de las universidades del mundo real (Bosch y Ferrer, 2002).

En líneas generales, se podría concluir que los resultados del análisis de las medidas recogidas en los ejes de participación política de las mujeres en las universidades españolas muestran una intención generalizada de conseguir la participación equilibrada, que requiere la Ley de Igualdad y la LOMLOU para lograr una mayor presencia en las estructuras de poder y acabar con la segregación horizontal y vertical.

En el contexto de los rápidos cambios de la sociedad que repercuten de manera invariable sobre la educación superior, los diversos países europeos han dado respuestas variadas a la necesidad generalizada de repensar las estructuras de gobierno de la universidad. El llamado proceso Bolonia (Espacio Europeo de Educación Superior: EEES) y sus efectos directos sobre el Sistema de Educación Superior hacen replantear las políticas básicas institucionales, para enfrentarse de manera más eficiente a los retos actuales de sus "clientes" y de la sociedad en general (Ceacero e Ion, 2011).

"Las organizaciones universitarias exitosas son aquellas que logran modificar el funcionamiento de su gobierno y sus formas de gestión para adaptarla a las cambiantes demandas del entorno en que se desenvuelven y a las transformaciones estructurales de aquél" (Brunner, 2011: 37). "El sistema universitario español demanda más calidad, más equidad y una relación más próxima con la sociedad, por lo que debemos adoptar políticas para que la ciudadanía ejerza su derecho a recibir una Educación Superior de calidad" (LOMLOU, 2007).

Abordar la igualdad desde el ámbito universitario resulta necesario pues en él siguen existiendo factores que pueden generar desigualdad y afectan e influyen en el desarrollo social, en la calidad de vida de las personas y en la gestión del conocimiento (creación y transmisión). La igualdad se introduce en las Universidades como parte de su compromiso social y constituye un factor de calidad y modernización, centrado en las personas, y en toda la actividad universitaria (Instituto Andaluz de la Mujer, 2011).

El proceso es lento, pero las medidas adoptadas, comunes y específicas, parecen denotar que el proceso está en marcha. Las universidades están sufriendo una remodelación importante para adecuarse al Espacio Europeo de Educación Superior y, en concreto, tener en cuenta el desarrollo del principio de igualdad recogido en el Real Decreto 1393/2007. Se plantea así la necesidad de proceder de un modo coordinado para incorporar la perspectiva de género, tanto en los estudios de grado como de posgrado, porque el conocimiento sin sesgo de género es esencial para el cambio de actitudes 
María del Carmen Díaz-Fernández, María del Rocío Martínez-Torres y Jesús Manuel López-Bonilla. Mujeres en órganos de gobierno universitarios. Nuevo contexto normativo y politicas de igualdad

sexistas y para la erradicación de comportamientos discriminatorios (TapiaHernández, 2009).

\section{Método}

Tras el análisis previo de la situación de las universidades españolas en materia de género (Martínez-Torres et al., 2010a) y de las políticas existentes en materias de igualdad en la Administración Pública y en las universidades españolas (Martínez Torres et al., 2010b), el propósito de este trabajo ha sido identificar las principales barreras que dificultan el acceso equitativo de las mujeres a los órganos de gobierno de las universidades españolas. Los resultados podrán servir como punto de partida para el diseño de políticas de igualdad real y efectiva. Para ello se ha empleado la técnica científica de elaboración de mapas conceptuales (Trochim, 1989).

Un mapa conceptual es una forma de conceptualización estructurada que puede ser usada por grupos para desarrollar esquemas conceptuales y guiar una evaluación o una planificación (Toral et al., 2007; Trochim, 1989). Para su elaboración, y a diferencia de otros autores como Novak y Gowin (1984), se considera un procedimiento científico que tiene en cuenta elementos cuantitativos y cualitativos.

La elaboración del mapa conceptual consta de las siguientes fases (Kolb y Shepherd, 1997): 1) selección y preparación de los participantes; 2) tormenta de ideas (conceptos); 3) estructuración y ponderación de las ideas; 4) representación de las ideas en un mapa conceptual, y 5) interpretación de los mapas.

Los participantes generan información a través de un brainstorming o tormenta de ideas. A continuación, los datos son estructurados y clasificados, utilizando un doble análisis estadístico. El primero, un escalamiento multidimensional, sitúa en el espacio (normalmente bidimensional) cada una de las ideas identificadas, representándolas mediante un punto. El segundo, un análisis clúster, toma como datos el resultado del análisis anterior y agrupa las ideas en conceptos homogéneos, representándolos en el espacio anterior.

El mapa conceptual resultante muestra las principales categorías matemáticas determinadas a partir de las ideas derivadas de los participantes; y se representa cada subconjunto de ideas en un mapa en forma de clúster. Este procedimiento recae sobre la base de opiniones de expertos, pero con un tratamiento estadístico de datos recopilados que garantizan que sus conclusiones son el resultado de la conceptualización de la totalidad del grupo y no de cada individuo a nivel particular (Toral et al., 2006). 


\section{Selección y preparación de los participantes}

Una de las tareas más importantes en el desarrollo de un mapa conceptual es decidir quién participará en el proceso. La experiencia muestra que la conceptualización es mejor cuando el proceso incluye un amplio rango de expertos (Martínez-Torres et al., 2005), pues, además de ayudar a asegurar que los diferentes puntos de vista serán considerados, fomenta la construcción correcta del mapa conceptual.

Para este estudio se contó con personal de la universidad correspondiente a los colectivos PDI, PAS y Alumnado, y con una persona externa a la institución. Todos con experiencia contrastada tanto en la universidad como en temas de género. La participación de los tres primeros ha sido proporcional a su representación en el Claustro (70\%, 20\% y 10\%, respectivamente), representando la persona externa su homónimo en el Consejo Social. En total fueron 20 participantes, número que está dentro de los límites, entre 10 y 20, establecidos por Trochim (1989).

\section{Tormenta de ideas}

En esta fase se pide a los participantes que identifiquen las dificultades encontradas por las mujeres para acceder a los órganos de gobierno universitarios, usando para ello la técnica del brainstorming o tormenta de ideas. Se identificaron 54 ideas (véase Tabla 1). ${ }^{2}$

\section{Estructuración y ponderación de las ideas}

Tras tener el conjunto de ideas que describen el dominio conceptual para el tema, durante esta etapa es necesario realizar dos tipos de tareas. En primer lugar, cada participante establece tanto el número de agrupaciones como las ideas incluidas en las mismas. El resultado de esta tarea es una Matriz Individual de Similitud (Snxn), que recoge los pares de ideas agrupadas conjuntamente en una matriz binaria en la cual el valor " 1 " indica que el par de ideas fue colocado por el participante en la misma agrupación; en tanto que el "0", refleja el fenómeno contrario. Sumando las matrices individuales de todos los participantes, se obtiene la Matriz Total ( $\operatorname{Tn} x n)$, que nos servirá como punto de partida para el análisis estadístico posterior.

2 Las tablas y la figura se encuentran en el Anexo, al final del presente artículo (Nota del editor). 
La segunda de las tareas consistió en ponderar cada idea en función de la dificultad que supusiese acceder a un órgano de decisión. Se empleó para ello una escala tipo Likert 1-5, en la cual $1=$ "poca dificultad" y $5=$ "mucha dificultad". El valor $0=$ "ninguna dificultad" no se tuvo en cuenta, dado que en el brainstorming se pidió explícitamente que se identificaran dificultades que se percibieran para el acceso a los órganos de gestión, ya fuese en mayor o menor medida.

\section{Representación de las ideas en forma de mapa conceptual}

A partir de los datos obtenidos en la fase anterior, se realiza un doble análisis de los mismos: escalamiento multidimensional y análisis clúster. El escalamiento multidimensional es una herramienta matemática que utiliza distancias entre objetos, sujetos o estímulos para producir una representación espacial de los mismos. En el mapa conceptual, este escalamiento multidimensional sienta las bases de un mapa de puntos que representa el conjunto de declaraciones hechas durante la sesión de brainstorming, partiendo de la matriz de similitud o de distancias de las ideas seleccionadas. El método utilizado con mayor frecuencia para determinar las coordenadas de cada objeto es un proceso iterativo comúnmente referenciado como Algoritmo de Shepard-Kruskal (Fahrmeir y Hamerle, 1984).

El escalamiento multidimensional proporciona el mapa de puntos de la Figura 1. En ésta cada punto representado mediante la imagen $\bigcirc$ representa cada una de las 54 ideas de la Tabla 1. La distancia entre los puntos indica la similitud o semejanza entre las mismas. Aquellos puntos más cercanos son más semejantes, mientras que los más alejados son conceptualmente más dispares entre sí (véase la Figura 1).

Una vez obtenida la representación bidimensional de las 54 ideas, es necesario clasificarlas en grupos homogéneos para identificar las dificultades de acceso a los órganos de gobierno en las universidades españolas. Esta categorización se lleva a cabo a través de un análisis clúster, que organiza la información proveniente del escalamiento multidimensional y no de la Matriz de Similitud (Everitt, 1993). Se utiliza el algoritmo de Ward (1963) para ello. Los resultados obtenidos en este análisis se muestran en la Figura 1 a partir de los siete clústers representados.

Los clústers identificados representan el mayor orden de agrupaciones conceptuales que se pueden presentar, desarrolladas por los participantes en relación con el tema a tratar. Además de esta clasificación recogida en la Figura 1, en el análisis se incluyen las ponderaciones medias de cada idea 
otorgadas por los participantes, poniéndose por tanto de relieve en sus resultados la importancia relativa de cada ítem, en relación con la dificultad de acceso de la mujer en los órganos de gobierno universitarios.

\section{Análisis de frabilidad}

Con el propósito de garantizar los resultados obtenidos, se realizó un análisis de fiabilidad. La teoría sobre fiabilidad usada de manera tradicional en la investigación de las ciencias sociales no encaja apropiadamente en los mapas conceptuales, porque asume una respuesta correcta para cada ítem testado que es conocido a priori. Además, el resultado individual de cada ítem es catalogado como correcto o incorrecto.

Sin embargo, este procedimiento no se puede aplicar para los mapas conceptuales, donde la respuesta para cada ítem no puede ser considerado en términos simples (correcto o incorrecto). Por esta razón, la estructura de la matriz de datos es invertida (comparada a la teoría tradicional) para la medición de la fiabilidad. Así, por ejemplo, los participantes son ubicados en columnas y los ítems (o pares de ítems) son localizados en filas.

La medición de la fiabilidad se centra en la consistencia del grupo teóricamente homogéneo de participantes. Para esta investigación, eso llega a ser útil cuando se considera la fiabilidad de la matriz de similitud o la fiabilidad del mapa resultante de ideas individuales (Trochim, 1993). El resultado clave de la técnica del mapa conceptual es el mapa de dos dimensiones. Consecuentemente, los esfuerzos de medición de la fiabilidad son centrados sobre la etapa central del análisis: el desarrollo y representación de estos mapas.

En el estudio publicado por Trochim (1993), la fiabilidad del mapa conceptual fue validada por seis coeficientes, que podían ser estimados a partir de datos posibles sobre algún proyecto de mapa conceptual. Estos coeficientes fueron definidos y estimados para 38 proyectos de mapas conceptuales. Los resultados muestran que un proceso de mapa conceptual puede ser fiable acorde a estándares generalmente reconocidos para determinados niveles de fiabilidad.

Todos los indicadores de fiabilidad usados por Trochim (1993) son considerados para nuestro estudio y sirvieron para comparar nuestros resultados con los alcanzados por este autor en sus investigaciones (véase Tabla 2).

Los resultados recogidos en la Tabla 2 hacen evidente el alto grado de fiabilidad encontrado en el mapa conceptual propuesto, al estar todos los indicadores incluidos dentro de los rangos usuales establecidos por Trochim (1989). 
María del Carmen Díaz-Fernández, María del Rocío Martínez-Torres y Jesús Manuel López-Bonilla. Mujeres en órganos de gobierno universitarios. Nuevo contexto normativo y politicas de igualdad

\section{Resultados}

\section{Interpretación de los mapas}

Para poder interpretar los mapas se organizó un grupo de trabajo final. Por lo general, los resultados derivados del análisis clúster son más difíciles de interpretar que aquellos que emanan del escalamiento multidimensional. El análisis clúster es visto como un indicador, y la clave está en el mantenimiento de la integridad de los resultados del escalamiento multidimensional, proporcionando una solución que no permitirá la superposición entre los clústers. A la hora de establecer el nombre de los diferentes clústers se recurrió al consenso, y se eligió el definitivo de entre los diferentes propuestos por los grupos de participantes.

Así, las técnicas empleadas permiten agrupar, en primer lugar, las 54 ideas consideradas en siete grupos homogéneos o clústers de ideas similares, y en segundo lugar, atendiendo a esta similitud, los clústers en cuatro grandes regiones de ideas homogéneas o similares (véanse Figura 1 y Tabla 3 ).

La primera región, situada a la izquierda de la Figura 1, hace referencia a la difícil conciliación entre la vida laboral y familiar. Aglutina las ideas del clúster 1, en las que se hace notorio, además de consecuencias directas del techo de cemento (cargas familiares), elementos tradicionalmente socioculturales (mal visto que el hombre coja la baja maternal, priorización de la vida familiar, falta de delegación: “mamá lo hace todo”...) (véase Tabla 3).

Esta región y la 3 serán las de mayor peso/importancia otorgada por los participantes a este grupo de ideas; es decir, las que incluyen las barreras consideradas más importantes por parte estos individuos. Una vez más se hace evidente las consecuencias del lastre sociocultural históricamente constituido, dados los distintos roles que han tenido que cumplir mujeres y hombres, y la división sexual del trabajo.

La segunda región, localizada en la parte inferior de la Figura 1, engloba, catalogados en dos clústers, los obstáculos que tienen que superar las mujeres en los órganos de dirección. Así pues, en el clúster 2 se ubican los referentes al "techo de cristal" y en el clúster 6 los concernientes al acceso y promoción femenina en estos órganos.

En cuanto al peso/importancia, esta región le sigue a: la 1 (conciliación vida laboral y familiar) y a la 3 (situación patriarcal), ya comentadas en la Figura 1. Esto nos lleva a presuponer que, si bien los tradicionales lastres siguen teniendo importancia, empieza a emerger también una incipiente evolución en la mentalidad general de los colectivos implicados. La confirmación de 
esta suposición inicial reportaría lógica al menor peso otorgado a estos clústers de la segunda región.

No obstante, hemos de resaltar que junto a este resultado aparecen también sentimientos opuestos a esta evolución, como el asentimiento, manifestación de las nuevas dificultades a las que se enfrentan las mujeres cuando deciden acceder o promocionar en los órganos de decisión. Este asentimiento puede ser fruto quizás del elevado peso aún de los obstáculos de la región 3 y la dificultad de combatir los de la región 1 por parte del colectivo femenino.

La tercera región, localizada en la parte alta de la Figura 1, abarca un único clúster, denominado "sistema patriarcal". Esta región es, junto con la primera, la que ostenta el mayor peso o grado de importancia en el mapa conceptual realizado. Considerando que el peso relativo de cada clúster podría ser usado como un punto inicial, cuando tenemos que decidir las líneas de acción o medidas estratégicas por abordar en relación con la elaboración, desarrollo y promulgación de las políticas de igualdad de género en la universidad, estos hallazgos podrían ser de gran utilidad a la hora de subsanar estas debilidades o carencias.

Es decir, en virtud de estos hallazgos, habría que tener en cuenta en el diseño de políticas de igualdad de género una serie de medidas capaces de enfrentar carencias históricas y tradicionalmente asumidas y debatidas, muy presentes todavía, como: el elevado peso del sistema patriarcal y la dificultad de conciliación entre la vida familiar y laboral. Ello nos lleva también a demostrar la escasa eficacia práctica de las políticas actuales a la hora de subsanar estos problemas de igualdad de género.

La última región, localizada en la parte derecha de la Figura 1, representa la estructura de las relaciones de género, que son consideradas como las barreras percibidas que menos dificultan el acceso a los órganos de decisión. Este resultado es un reflejo contrastable en los hallazgos de la literatura de género, de la situación actual de la mujer en los órganos de gobierno.

Una segunda lectura de estos resultados nos lleva a desterrar algunas de las "tradicionales" justificaciones que han sido alegadas, en relación con la ausencia de la mujer en los órganos de decisión: los estereotipos de género, en último lugar, y la ausencia de liderazgo femenino, una vez más, parecen no tener gran importancia en este contexto.

Cabe destacar, en virtud de los resultados alcanzados, cómo las universidades, en materia de política universitaria, en aras a alcanzar una igualdad efectiva y equitativa entre los géneros, debería acometer líneas de acción estratégica tendentes a subsanar las debilidades que esta situación provoca y es 
promovida, en una gran parte, por las siguientes causas: 1) la conciliación de la vida laboral y familiar; 2) el sistema patriarcal; 3) el techo de cristal; 4) la masculinización del puesto; 5) el acceso y la promoción; 6) la ausencia de liderazgo femenino; y 7) los estereotipos de género.

\section{Conclusiones}

Desde hace más de 25 años, el tema de la presencia de las mujeres o de sus necesidades en las políticas públicas ha sido objeto de un intenso debate por parte de diferentes actores, tanto en las sociedades nacionales como en el ambiente internacional, en especial en los organismos multilaterales. En este proceso, ampliamente analizado en sus características, instrumentos, debilidades, fortalezas y consecuencias, han aflorado paradigmas a través de los cuales se ha tratado de dar respuesta a la desigualdad de género existente. Y es que, tal y como apunta Sánchez (2002: 342-370), el contexto normativo actual ha dado lugar a un cambio en la teoría feminista; sus argumentos se han desplazado desde posiciones universalistas hacia las que abogan por las diferencias.

Las mujeres han estado presentes siempre de alguna manera en las estrategias y políticas de desarrollo, aun cuando hasta hace muy poco tiempo esa presencia era apenas perceptible, muy opaca y hasta irreal (García, 2008). Las diferencias entre géneros, esencialmente sociales y culturales e históricamente constituidas, a causa de los distintos roles que han tenido que cumplir mujeres y hombres, y la división sexual del trabajo, han sido un importante lastre que las mujeres han tenido y tienen que soportar.

Un amplio elenco de tratados, leyes y jurisprudencia en general, tanto a nivel nacional, europeo como internacional, desde la Carta de San Francisco redactada en el Acto de Constitución de Naciones Unidas en 1945, han tenido como objeto garantizar esta igualdad entre mujeres y hombres, marcando el devenir de los avances políticos y legales en esta materia, además de impregnarles consistencia, fortaleza y obligatoriedad de cumplimiento.

En virtud de estos avances y logros, el principio de igualdad inicial de derecho o de jure y la igualdad de hecho o de facto pasaría a una igualdad de oportunidades, una igualdad de trato y una igualdad de resultados; la igualdad de oportunidades deja de ser una opción para convertirse en una obligación de los Estados.

En este contexto se enmarca el estudio que presentamos. En él se han identificado las dificultades de acceso de las mujeres a los órganos de gobierno de las universidades españolas, mediante la aplicación de mapas 
conceptuales (Trochim, 1989). Los resultados alcanzados podrían servir como punto de partida para diseñar las líneas estratégicas que guíen el diseño de políticas de igualdad.

El tema de las políticas en las estrategias de institucionalización del enfoque de género en las organizaciones del Estado es de importancia primordial por el papel que éstas cumplen en todo el proceso de planificación o diseño de las acciones públicas, ya que son la columna vertebral sobre la cual se construye una estrategia eficiente.

Estas políticas proveen de conceptos y objetivos a los planes, y éstos, a su vez, de conceptos y estrategias a los programas y proyectos que se ejecutan. En la medida como las políticas sean correctamente formuladas, su desarrollo en otros instrumentos de menor jerarquía que orientan las acciones será más eficiente.

En virtud de los hallazgos alcanzados y presentados, las líneas estratégicas de las distintas políticas de igualdad deberían hacer especial énfasis en la subsanación de las siguientes dificultades, siguiendo este orden de prelación: 1) la conciliación de la vida laboral y familiar; 2) el sistema patriarcal; 3) el techo de cristal; 4) la masculinización del puesto; 5) el acceso y la promoción; 6) la ausencia de liderazgo femenino; y 7) los estereotipos de género.

Si bien la elevada fiabilidad contrastada obtenida en el estudio es garante de la veracidad de los resultados presentados, existen limitaciones referidas a su carácter sectorial (sólo en el ámbito de la universidad española) que requieren tomar con la debida precaución estos hallazgos. Otra limitación se refiere al hecho de representar las ideas en un espacio bidimensional. Sin embargo, esta limitación se ve compensada por la mayor facilidad en la interpretación de los resultados, hecho que no se tendría haciendo uso de espacios de mayor dimensión.

Como continuación de esta investigación se propone contrastar los resultados obtenidos con los distintos planes de igualdad diseñados en la actualidad en las universidades españolas, con el fin de detectar en qué medida dichos planes dan respuesta a las dificultades que realmente existen para la consecución de esta igualdad.

La investigación más reciente ha demostrado que en muchos campos del conocimiento científico las variables de género no son consideradas todavía suficientemente. Perviven estereotipos y sesgos en la investigación y en los desarrollos tecnológicos que, en muchas ocasiones, construyen realidades generalmente aceptadas (Ministerio de Ciencia e Innovación, 2011). 
María del Carmen Díaz-Fernández, María del Rocío Martínez-Torres y Jesús Manuel López-Bonilla. Mujeres en órganos de gobierno universitarios. Nuevo contexto normativo y politicas de igualdad

\section{Agradecimiento}

Los autores de este trabajo queremos agradecer tanto los comentarios de los dictaminadores como la gran labor del equipo editorial de Convergencia. La ayuda de todos ellos no sólo ha hecho posible la publicación de nuestro trabajo, sino que ha contribuido, sin lugar a dudas, a incrementar el valor del mismo.

\section{Referencias}

Alonso-Sánchez, María (2002), “Las Académicas. Profesorado Universitario y Género", en Revista de Educación, núm. 328, España: Ministerio de Educación, Cultura y Deporte.

Archenti, Nélida y Albaine, Laura (2014), "Los desafíos de la paridad de género. Tensión normativa y violencia política en Bolivia y Ecuador", en Revista Punto Género, núm. 3 , Chile: Universidad de Chile.

Arreman, Inger y Weiner, Gaby (2007), "Gender, Research and Change in Teacher Education: a Swedish Dimension”, en Gender and Education, vol. 19, núm. 3, Inglaterra: Routledge Journals, Taylor \& Francis.

Bosch, Esperanza y Ferrer, Victoria (2002), "El feminismo académico y su difusión social: nuestra experiencia desde la Universidad de las Islas Baleares", en Caporale, S. et al. [eds.], La difusión del conocimiento en los estudios de las mujeres. Dinámica y estrategias depoder y ciudadania, Actas del II Congreso Internacional de AUDEM. Disponible en http: www.espagrafic.com [17 de enero de 2003].

Bourdieu, Pierre (2000), La dominación masculina, España: Anagrama.

Broadbridge, Adelina y Fielden, Sandra (2015), "Handbook of Gendered Careers", en Management: Getting In, Getting On, Getting Out, Estados Unidos: Edward Elgar Publishing.

Brunner, José Joaquín (2011), “Gobernanza universitaria: tipología, dinámicas y tendencias", en Revista de Educación, núm. 355, España: Ministerio de Educación, Cultura y Deporte.

Ceacero, Diego e Ion, Giorgeta (2011), "Dilemas en el Gobierno de las Universidades Españolas: Autonomía, Estructura, Participación y Desconcentración”, en Revista de Educación, núm. 355, España: Ministerio de Educación, Cultura y Deporte.

Clegg, S. (2008), "Academic Identities Under Threat?", en British Educational Research Journal, vol. 34, núm. 3, Gran Bretaña.

Clegg, Sue (2008), "Academic Identities Under Threat?", en British Educational Research Journal, vol. 34, núm 3, Inglaterra: Wiley-Blackwell.

Donoso, T. et al. (2011), "Barreras de Género en el Desarrollo Profesional de la Mujer Universitaria”, en Revista de Educación, vol. 355, España: Ministerio de Educación, Cultura y Deporte.

Eisenmann, Linda (2003), "Gender and The Restructured University: Changing Management and Culture in Higher Education", en Review of Higher Education, vol. 26, núm. 4, Estados Unidos: Johns Hopkins University Press. 
Convergencia Revista de Ciencias Sociales, núm. 75, 2017, Universidad Autónoma del Estado de México

Everitt, Brian (1993), Cluster Analysis, Estados Unidos: Halsted.

Fahrmeir, L. y Hamerle, A. (1984), Multivariate Statistiche Verfahren, Alemania: de Gruyter. Frundt, Henry (2002), "Central American Unions in The Era of Globalization”, en Latin American Research Review, vol. 37, núm. 3, Estados Unidos: Latin American Studies Association.

García, Evangelina (2003), Hacia la Institucionalización del Enfoque de Género en Politicas Públicas, Venezuela: Fundación Friedrich Ebert.

García, Evangelina (2008), "Políticas de Igualdad, Equidad y Gender, Mainstreaming. ¿De qué estamos hablando?”, en Marco Conceptual, Programa de las Naciones Unidas para el Desarrollo (PNUD), El Salvador: PNUD.

Gisbert, Marina (2007), "Mujer y Sociedad: Evolución de la Mujer en la Sociedad y en el Mundo Laboral en el Siglo XX. Realidad Actual de la Mujer en España”, en Mujer y Trabajo en el Siglo XXI: Estudio y Prevención de Riesgos Laborales, España: Universidad Internacional Menéndez Pelayo.

Grueso, Merlin (2010), “Implementación de buenas prácticas de promoción de personal y su relación con la cultura y el compromiso con la organización”, en Innovar, vol. 20, núm. 36, Colombia: Universidad Nacional de Colombia.

Guerrero, Antonio (2007), "La Doble Contribución de la Sociología a la Formación del Profesorado”, en Revista Internacional de Sociologia (RIS), vol. 48. España: Consejo Superior de Investigaciones Científicas.

Ibáñez, Marta (2010), “Al Otro Lado de la Segregación Ocupacional por Sexo. Hombres en Ocupaciones Femeninas y Mujeres en Ocupaciones Masculinas", en Revista Internacional de Sociología (RIS), vol. 68, núm. 1, España: Consejo Superior de Investigaciones Científicas.

Instituto Andaluz de la Mujer (2011), La Igualdad entre Mujeres y Hombres en las Universidades a partir del Diagnóstico y los Planes de Igualdad, España: Consejería para la Igualdad y Bienestar Social, Instituto Andaluz de la Mujer.

Jalalzai, Farida (2008), “Women Rule: Shattering the Executive Glass Ceiling”, en Politics and Gender, vol. 4, núm. 2, Estados Unidos: Cambridge University Press.

Kolb, Darl y Shepherd, Deborah (1997), “Concept Mapping Organizational Cultures”, en Journal Management Inquiry, vol. 6, núm. 4, Estados Unidos: Sage Publications.

Lambert, Cath y Parker, Andrew (2006), "Imagination, Hope and the Positive Face of Feminism: Pro/Feminist Pedagogy in 'Post' Feminist Times?”, en Studies in Higher Education, vol. 31, núm. 4, Inglaterra: Routledge Journals, Taylor \& Francis LTD.

López-Bonilla, Jesús et al. (2014), "Diferencias de género en las universidades españolas y en sus órganos gerenciales”, en Revista Venezolana de Gerencia, vol. 19, núm. 66, Venezuela: Universidad del Zulia.

LOMLOU (Ley Orgánica de Modificación de la Ley Orgánica de Universidades) (2007), Ley Orgánica 4/2007 de Modificación de la Ley Orgánica de Universidades, en BOE núm. 89, [13 de abril de 2007].

Lovell, Peggy (2006), “Race, Gender, and Work in Sao Paulo, Brazil, 1960-2000”, en Latin American Research Review, vol. 41, núm. 3, Estados Unidos: Latin American Studies Association.

Maldonado, María (2003), "A propósito de La dominación masculina de Pierre Bourdieu”, en Revista Sociedad y Economía, núm. 4, abril, Colombia: Universidad del Valle, Cali. 
María del Carmen Díaz-Fernández, María del Rocío Martínez-Torres y Jesús Manuel López-Bonilla. Mujeres en órganos de gobierno universitarios. Nuevo contexto normativo y politicas de igualdad

Marcenaro, Oscar et al. (2007), "El Éxito en la Universidad: Una Aproximación Cuantílica”, en Revista de Economía Aplicada, vol. 15, núm. 44, España: Universidad de Zaragoza.

Martín, Milagros y Díaz, María del Carmen (2010), "El rol de la mujer en la universidad: un estudio de género", en II Congreso Universitario Nacional "Investigación y Género", España: Universidad de Sevilla.

Martínez-Torres, María del Rocío et al. (2005), "A Digital Signal Processing Teaching Methodology Using Concept Mapping Techniques”, en IEEE Transaction on Education, vol. 48, núm. 3, Estados Unidos: IEEE-Inst Electrical Electronics Engineers.

Martínez-Torres, María del Rocío et al. (2010a), "Estudio de género en los órganos decisorios de la universidad”, en Vázquez Bermúdez, I. [coord.], Investigaciones Multidisciplinares en Género, II Congreso Universitario (Nacional) "Investigación y Género", España.

Martínez-Torres, María del Rocío et al. (2010b), "El Análisis de las Políticas de Igualdad en la Administración Pública”, en Vázquez Bermúdez, I. [coord.], Investigaciones Multidisciplinares en Género, II Congreso Universitario (Nacional) "Investigación y Género", España.

Mateos de Cabo, Ruth et al. (2010), "Discriminación en Consejos de Administración: Análisis e Implicaciones Económicas”, en Revista de Economía Aplicada, vol. 18, núm. 53, España: Universidad de Zaragoza.

Ministerio de Ciencia e Innovación (2011), Manual el Género en la Investigación, España: Ministerio de Ciencia e Innovación, Gobierno de España.

Morrison, Ann (1987), Breaking the Glass Ceiling, Estados Unidos: Addison-Wesley.

Novak, Joseph y Gowin, D. Bob (1984), Learning How to Learn, Estados Unidos: Cambridge University Press.

Ordorica-Mellado, Manuel (2014), “1974: momento crucial de la política de población”, en Papeles de Población, vol. 20, núm. 81, México: Universidad Autónoma Estado México.

Palma, Esperanza y Cerva, Daniela (2014), "La importancia de las instituciones y la organización de las mujeres. El cumplimiento de la cuota de género en las elecciones federales mexicanas de 2012”, en Politica y Gobierno, vol. 21, núm. 2, México: Centro de Investigación y Docencia Económicas.

Pearce, John y Zahra, Shaker (1992), "Board Composition from a Strategic Contingency Perpective”, en Journal of Management Studies, vol. 29, núm. 4, Inglaterra: WileyBlackwell.

Phelps, Edmund (1972), “The Statistical Theory of Racism and Sexism”, en The American Economic Review, vol. 62, núm. 4, Estados Unidos: American Economic Association.

PEIO (Plan Estratégico de Igualdad de Oportunidades 2014-2016), España: Instituto de la Mujer y para la Igualdad de Oportunidades y el Ministerio de Sanidad, Servicios Sociales e Igualdad de España. Disponible en: http://www.inmujer.gob.es/ [27 de marzo de 2017].

Priola, Vincenza (2007), "Being Female Doing Gender. Narratives of Women in Education Management”, en Gender and Education, vol. 19, núm. 1, Inglaterra: Routledge Journals, Taylor \& Francis.

Probert, Belinda (2005), “I just couldn't fit it in': Gender and Unequal Outcomes in Academic Careers", en Gender, Work and Organization, vol. 12, núm. 1, Inglaterra: Wiley-Blackwell.

Rees, Teresa (2001), "Mainstreaming Gender Equality in Science in the European Union", en Gender and Education, vol. 13, núm. 3, Inglaterra: Routledge Journals, Taylor \& Francis. 
Rodríguez-Enriquez, Corina (2015), “Economía feminista y economía del cuidado. Aportes conceptuales para el estudio de la desigualdad”, en Nueva Sociedad, núm. 256, Argentina.

Salinas, Paulina et al. (2010), "The female labor market: an empirical study of the mining region of Antofagasta, Chile”, en Innovar, vol. 20, núm. 38, Colombia: Universidad Nacional de Colombia.

Sánchez, Cristina (2002), “Feminismo y ciudadanía”, en Díaz, Elías y Colomer, José Luis [eds.], Estado, justicia, derechos, España: Alianza.

Subramaniam, Nava (2003), "Factors Affecting the Career Progress of Academic Accountants in Australia: Cross-Institutional and Gender Perspectives”, en Higher Education, vol. 46, núm. 4, Holanda: Springer.

Tapia-Hernández, Sara (2009), "Desvelar la discriminación de género mediante la actividad docente en un contexto universitario”, Tesis Doctoral, España: Universidad de Valladolid.

Timmers, Tanya M. et al. (2010), "Gender Diversity Policies in Universities: A MultiPerspective Framework of Policy Measures”, en Higher Education, vol. 59, núm. 6, Holanda: Springer.

Toral, Sergio et al. (2006), "Planning a Master's Level Curriculum According to Career Space Recommendations Using Concept Mapping Techniques", en International Journal of Technology and Design Education, vol. 16, núm. 3, Holanda: Springer.

Toral, Sergio et al. (2007), "An Electronic Engineering Curriculum Design Based on Concept-Mapping Techniques", en International Journal of Technology and Design Education, vol. 17, núm. 3, Holanda: Springer.

Trochim, William (1989), "An Introduction to Concept Mapping for Planning and Evaluation”, en Evaluation Program Planning, vol. 12, núm. 1, Estados Unidos: Pergamon-Elsevier Science.

Trochim, William (1993), “The Reliability of Concept Mapping”, en Annual Conference of the American Evaluation Association, Estados Unidos.

Villavicencio, Luis (2014), “Minorías étnico-culturales y derechos de las mujeres”, en Revista de Ciencia Política, vol. 34, núm. 3, Chile: Pontificia Universidad Católica de Chile, Instituto de Ciencia Política.

Ward, Joe (1963), "Hierarchical Grouping to Optimize an Objective Function", en Journal of American Statistical Association, vol. 58, núm. 301, Estados Unidos: American Statistical Association.

Wolfers, Justin (2006), "Diagnosing Discrimination: Stock Returns and Ceo Gender", en Journal of the European Economic Association, vol. 4, núm. 2-3, Inglaterra: WileyBlackwell. 
María del Carmen Díaz-Fernández, María del Rocío Martínez-Torres y Jesús Manuel López-Bonilla. Mujeres en órganos de gobierno universitarios. Nuevo contexto normativo y politicas de igualdad

\section{Anexo}

\section{Tabla 1}

\section{Ideas identificadas en la sesión de brainstorming}

\begin{tabular}{l}
\hline \multicolumn{1}{c}{ Ideas o Items } \\
\hline 1. Mentalidad muy masculina \\
\hline 2. Aspectos socioculturales \\
\hline 3. Techos de cristal \\
\hline 4. Techos de cemento (cargas familiares) \\
\hline 5. Conciliación vida familiar vida laboral \\
\hline 6. Aspectos educativos \\
\hline 7. Rol de cuidadoras \\
\hline 8. Miedo a la subordinación de una mujer \\
\hline 9. Roles de género \\
\hline
\end{tabular}

10. Falta de decisión a presentar candidaturas

11. Falta de confianza en conseguir el acceso

12. Puestos directivos con horarios tradicionales masculinos

13. Dificultad para conseguir los méritos

14. Cuestionamiento de los méritos

15. Permiso de maternidad

16. Maternidad (después del permiso)

17. Ausencia de normativa que garantice representación paritaria

18. Criterios de promoción asociados a cantidad y no a calidad

19. Rechazo a la mujer directiva por parte de las propias mujeres

20. Rechazo al abandono de conductas femeninas

21. Ausencia de ambiciones directivas

22. Escasez de referentes femeninos en puestos directivos

23. Desinformación de la cultura organizativa

24. Desinformación de los mecanismos de promoción y acceso

25. Priorización de la vida familiar frente a la laboral

26. Puestos directivos asociados a carrera docente-investigadora

27. Trabas por sentido de exclusividad de mujeres directivas 
28. Entornos de mayoritaria presencia masculina

29. Puestos ya ocupados por hombres

30. Mayor nivel de exigencia a las mujeres

31. Mayor esfuerzo para llegar y mantenerse en puesto directivo

32. Ausencia mujeres reuniones informales por cargas familiares

33. Falta de capacidad de delegación de las cargas familiares

34. Desconexión de redes informales-información clave

35. Edad como penalización en el acceso a puestos directivos

36. Universidad con liderazgo de orientación más tradicional

37. Falta de obligatoriedad de la baja paternal

38. Falta de apoyo del entorno para el acceso a puestos directivos

39. Escasez de tutorización a mujeres

40. Falta de empatía entre géneros

41. Miedo a habladurías por apoyar a mujeres

42. Escasez en rotación en puestos directivos

43. Miedo al absentismo laboral de la mujer

44. Cuestionamiento de la profesionalización de la mujer

45. Dificultad para obtener algunos méritos (estancias en el extranjero)

46. Culturas tradicionalmente machistas

47. Mayor peso de decisiones masculinas

48. Educación patriarcal

49. Escasez de información sobre acceso a la vida profesional universitaria

50. Falta de transparencia en la información sobre la promoción-ascenso

51. Competitividad en el acceso a puestos directivos

52. Camaradería entre hombres

53. Rivalidad entre mujeres

54. Falta de concienciación de la problemática de la dificultad existente para el acceso de las mujeres

Fuente: Elaboración propia. 
María del Carmen Díaz-Fernández, María del Rocío Martínez-Torres y Jesús Manuel López-Bonilla. Mujeres en órganos de gobierno universitarios. Nuevo contexto normativo y politicas de igualdad

\section{Tabla 2}

\section{Estadísticos descriptivos para la estimación de la fiabilidad}

\begin{tabular}{lcccccc} 
& $\mathrm{r}_{\mathrm{II}}$ & $\mathrm{r}_{\mathrm{IT}}$ & $\mathrm{r}_{\mathrm{IM}}$ & $\mathrm{r}_{\mathrm{RR}}$ & $\mathrm{r}_{\mathrm{SHT}}$ & $\mathrm{r}_{\text {SHM }}$ \\
\hline Número de proyectos & 33 & 33 & 33 & 37 & 33 & 33 \\
\hline Media & 0.81507 & 0.92965 & 0.86371 & 0.78374 & 0.83330 & 0.55172 \\
\hline Mediana & 0.82060 & 0.93070 & 0.86280 & 0.82120 & 0.84888 & 0.55881 \\
\hline Mínimo & 0.67040 & 0.88230 & 0.74030 & 0.42700 & 0.72493 & 0.25948 \\
\hline Máximo & 0.93400 & 0.97370 & 0.95490 & 0.93540 & 0.93269 & 0.90722 \\
\hline Desviación típica & 0.07016 & 0.02207 & 0.04771 & 0.12125 & 0.05485 & 0.15579 \\
\hline Nuestro mapa & 0.73399 & 0.92615 & 0.87592 & 0.7785 & 0.90652 & 0.83778 \\
\hline
\end{tabular}

Fuente: Elaboración propia.

\section{Tabla 3}

\section{Regiones}

\begin{tabular}{|c|c|c|}
\hline Región & Clúster & Ideas o Ítems \\
\hline $\begin{array}{l}\text { R1. Conciliación } \\
\text { vida familiar/ } \\
\text { laboral }\end{array}$ & $\begin{array}{l}\text { Clúster 1. Conciliación vida laboral/ } \\
\text { familiar }\end{array}$ & $4,5,15,16,25,32,33,37$ \\
\hline \multirow{2}{*}{$\begin{array}{l}\text { R2. Techo de } \\
\text { cristal }\end{array}$} & Clúster 2. Techo de cristal & $3,27,34,43,54$ \\
\hline & Clúster 6. Acceso y promoción & $\begin{array}{l}13,17,18,23,24,26,39 \\
42,45,49,50,51\end{array}$ \\
\hline $\begin{array}{l}\text { R3. Problemas } \\
\text { culturales }\end{array}$ & Clúster 3. Sistema patriarcal & $1,2,6,7,9,46,48$ \\
\hline \multirow{3}{*}{$\begin{array}{l}\text { R4. Estructura } \\
\text { de las relaciones } \\
\text { de género }\end{array}$} & Clúster 4. Estereotipos de género & $8,19,20,40,41,47,52,53$ \\
\hline & Clúster 5. Ausencia de liderazgo femenino & $10,11,14,21,22,31,35$ \\
\hline & Clúster 7. Masculinización del puesto & $12,28,29,30,36,38,44$ \\
\hline
\end{tabular}

Fuente: Elaboración propia. 


\section{Figura 1}

\section{Mapa conceptual: Ideas, clúster y regiones}
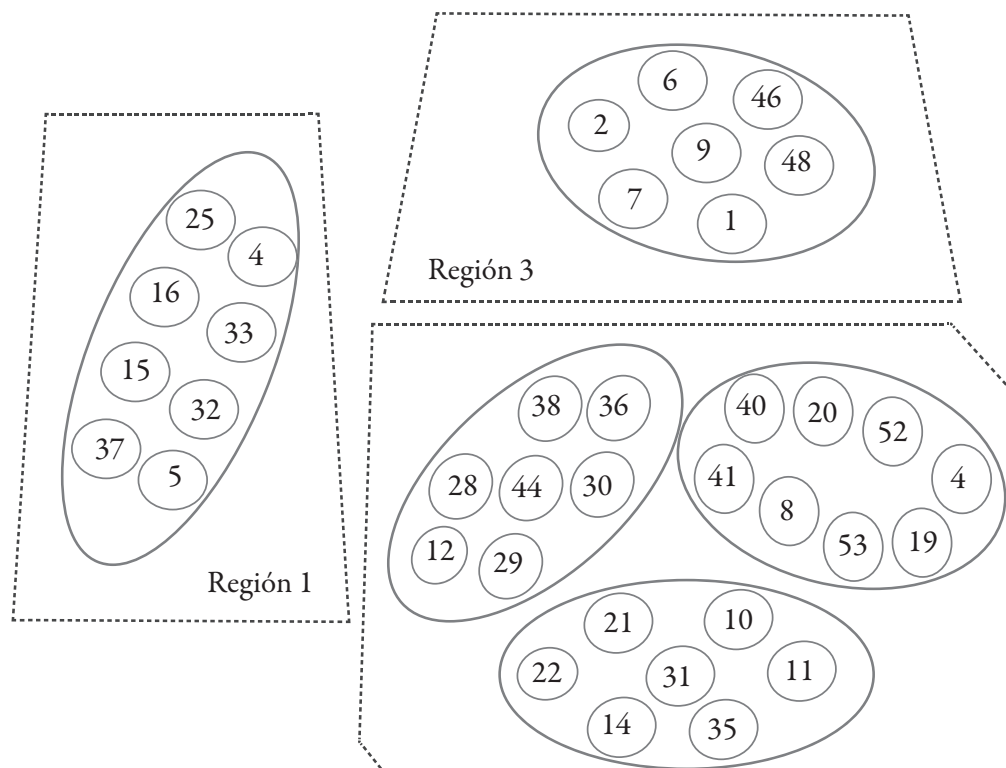

Región 4

Región 2
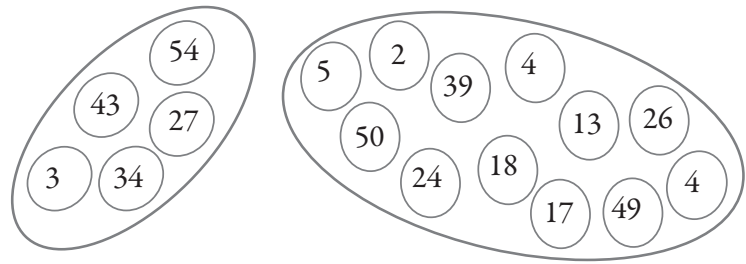

Fuente: Elaboración propia. 
María del Carmen Díaz-Fernández. Doctora en Administración y Dirección de Empresas, Profesora Contratada Doctora (Universidad de Sevilla) en el Departamento de Administración de Empresas y Comercialización e Investigación de Mercados (Marketing) (Facultad de Turismo y Finanzas). Grupo de Investigación: SEJ548: Big Data and Business Intelligence in Social Media. Publicaciones recientes: López-Bonilla, J. Manuel; MartínezTorres, M. Rocío y Díaz-Fernández, M. Carmen, "Diferencias de género en las universidades españolas y en sus órganos gerenciales", en Revista Venezolana de Gerencia, vol. 19, núm. 66 (2014); Martínez-Torres, M. Rocío y Díaz-Fernández, M. Carmen, "Current Issues and Research Trends on Open Source Software Communities”, en Technology analysis \& strategic management, vol. 26, núm. 1 (2014).

María del Rocío Martínez-Torres. Doctora en Administración y Dirección de Empresas y Profesora Titular de Universidad (Universidad de Sevilla) en el Departamento de Administración de Empresas y Comercialización e Investigación de Mercados (Marketing) (Facultad de Turismo y Finanzas). Grupo de Investigación: SEJ548: Big Data and Business Intelligence in Social Media. Publicaciones recientes: López-Bonilla, J. Manuel; MartínezTorres, M. Rocío y Díaz-Fernández, M. Carmen, "Diferencias de género en las universidades españolas y en sus órganos gerenciales", en Revista Venezolana de Gerencia, vol. 19, núm. 66 (2014); Martínez-Torres, M. Rocío y Díaz-Fernández, M. Carmen, "Current Issues and Research Trends on Open Source Software Communities”, en Technology analysis \& strategic management, vol. 26, núm. 1 (2014).

Jesús Manuel López-Bonilla. Doctor en Ciencias Económicas y Empresariales y Profesor Titular de Universidad (Universidad de Sevilla) en el Departamento de Administración de Empresas y Comercialización e Investigación de Mercados (Marketing) (Facultad de Ciencias Económicas y Empresariales). Grupo de investigación: SEJ480: Marketing, Innovación, Turismo y Sostenibilidad (Mitus). Publicaciones: López-Bonilla, J. Manuel; Martínez-Torres, M. Rocío y Díaz-Fernández, M. Carmen, "Diferencias de género en las universidades españolas y en sus órganos gerenciales", en Revista Venezolana de Gerencia, vol. 19, núm. 66 (2014); López-Bonilla, J. Manuel y López-Bonilla, L. Miguel, "Self-Service Technology Versus Traditional Service: Examining Cognitive Factors in the Purchase of the Airline Ticket", en Journal of Travel and Tourism Marketing, vol. 30, núm. 5 (2013). 
